\title{
Preliminary clinical outcomes of patients treated with vaginal brachytherapy alone using multi-channel vaginal brachytherapy applicator in operated early-stage endometrial cancer
}

\author{
Karthik S. Rishi ${ }^{1}$, Savitha David ${ }^{1}$, Muddappa Pathikonda ${ }^{1}$, Prakash Ramachandra ${ }^{1}$, Giri G.V. ${ }^{1}$, \\ Annapurna Vadaparty ${ }^{2}$, Srinath B.S. ${ }^{3}$ \\ ${ }^{1}$ Department of Radiation Oncology, Sri Shankara Cancer Hospital and Research Center, Bangalore, India \\ ${ }^{2}$ Department of Gynecologic Oncology, Sri Shankara Cancer Hospital and Research Center, Bangalore, India \\ ${ }^{3}$ Department of Surgical Oncology, Sri Shankara Cancer Hospital and Research Center, Bangalore, India
}

\begin{abstract}
Background: Recommendations for adjuvant treatment for postoperative, early-stage endometrial cancer varies from observation through vaginal brachytherapy alone to pelvic radiation. While observation alone can lead to recurrence, external radiotherapy has increased morbidity. The aim of this study is to show our results with vaginal brachytherapy alone using a multichannel applicator for treatment of early-stage endometrial cancer.

Materials and methods: Consecutive patients undergoing vaginal brachytherapy alone following surgery for early-stage endometrial cancer were examined. A Miami multichannel vaginal brachytherapy applicator was used to deliver HDR brachytherapy in 62 patients from May 2013 to June 2018. CT scan-based images guided planning. A dose of 5.5-6.5 Gy $\times 4$ fractions was prescribed $5 \mathrm{~mm}$ from the surface of the applicator.

Results: At a median follow up of 19 months (6-48 months), $93 \%$ of patients treated were alive with no recurrence. Two patients had only local recurrence, and 1 was salvaged with external radiotherapy and chemotherapy. There was only one nodal failure and 2 distant failures. There was no grade 2 or higher vaginal, gastrointestinal or genitourinary toxicity.

Conclusion: Vaginal brachytherapy alone using a multichannel applicator can be considered for early-stage endometrial cancers without compromising outcomes.

Key words: endometrium; brachytherapy; multichannel brachytherapy applicator

Rep Pract Oncol Radiother 2021;26(1):43-49
\end{abstract}

\section{Introduction}

Endometrial carcinoma is not uncommon among women in India. It is the fourth most common cancer in the United States, and the incidence is rising [1]. The same trend is also seen in urban India. Lifestyle factors, such as obesity and women favoring fewer children and delaying childbirth to later in life, are the main factors attributed to rising incidence [2]. Fortunately, most of these patients present in an early stage, and the outcomes with treatment are good.

The standard treatment for early-stage endometrial carcinoma is radical hysterectomy followed by

Address for correspondence: Dr Karthik S. Rishi, Department Of Radiation Oncology, Sri Shankara Cancer Hospital and Research Center, 1 St Cross, Shankarapuram, Basavanagudi, Bangalore - 560004, Karnataka, India, tel: (+91) 80 26981016, mobile: (+91) 9900101471;

e-mail: Drkarthiksrishi@gmail.com

This article is available in open access under Creative Common Attribution-Non-Commercial-No Derivatives 4.0 International (CC BY-NC-ND 4.0) license, allowing to download articles and share them with others as long as they credit the authors and the publisher, but without permission to change them in any way or use them commercially 
adjuvant radiotherapy. For stage I disease, the fiveyear survival is approximately $80-90 \%$ with standard treatment. Adjuvant treatment is based on risk stratification, which in turn is based on risk of disease recurrence characterized mainly by postoperative histopathological stage and grade of the disease. Low-risk disease includes grade 1 endometrial cancer with disease confined to the endometrium only and endometroid histology. No adjuvant treatment is recommended for these patients [3]. The PostOperative Radiation Therapy in Endometrial Cancer [PORTEC], GOG 99 and the ASTEC/EN.5 trials defined adjuvant treatment for intermediate and high-risk endometrial cancers [4-6]. High and intermediate risk are defined in PORTEC- 1 as women aged $>60$ years with disease involving the outer half of the myometrium and grade 3 histology. In the PORTEC-1 trial, women who underwent observation had a higher rate of relapse in the pelvis if these criteria were met. Patients were classified as having high-risk endometrial cancer if they had any of the following: serous adenocarcinoma (any stage), clear cell adenocarcinoma (any stage), or grade 3 deeply invasive endometrioid carcinoma [4-7].

The PORTEC- 2 trial looked at the need for external beam radiotherapy in high- intermediate-risk endometrial cancer. The trial established that vaginal brachytherapy alone was effective in ensuring vaginal control, with fewer toxic gastrointestinal effects than with EBRT [8]. Following the results of this trial, there has been a trend to avoid external beam radiotherapy in intermediate-risk endometrial cancer [9-11].

The aim of this study is to show our results with vaginal brachytherapy alone using a multichannel applicator for treatment of early-stage endometrial cancer.

\section{Materials and methods}

This study was a single institution, retrospective, nonrandomized study. All patients treated with vaginal brachytherapy alone from May 2013 to June 2018 were included in the analyses after obtaining approval from the IRB. Inclusion criteria were biopsy-proven, operated endometrial carcinoma patients in stages I and II of any histology. Patients who received external beam radiation or neoadjuvant chemotherapy were excluded from the analysis. All patients underwent either total abdominal hysterectomy with bilateral salpingo-oophorectomy with pelvic lymph node dissection or total abdominal hysterectomy only.

\section{Image registration}

Patients were planned for vaginal brachytherapy six weeks after surgery to allow for healing of the surgical wound. A Miami multichannel vaginal brachytherapy applicator (Varian medical systems) was used for all patients. The applicator is CT-compatible and has a $22 \mathrm{~mm}$ diameter applicator body with 6 channels positioned around the obturator. Centrally, there is a provision for a tandem to be placed if required. The obturator sleeves are available in diameters of $30 \mathrm{~mm}, 35 \mathrm{~mm}$ and $40 \mathrm{~mm}$ to suit the patient's needs (Fig. 1).

Application was done as an outpatient procedure. A vaginal examination was done before insertion to assess the size of the obturator sleeve required. The appropriate size of the applicator was inserted in the vagina and pushed inside until the patient winced. The applicator was held in place by an abdomen tie. For the first application, a noncontrast simulation CT scan with the applicator in situ was obtained. A fiducial marker was placed at the introitus, and $3 \mathrm{~mm}$ slice images were obtained from L5 to the upper femur on a 16-slice, $80 \mathrm{~cm}$ bore diameter Phillips CT scanner. The upper and lower levels of the CT scan were chosen for uniformity and reproducibility.

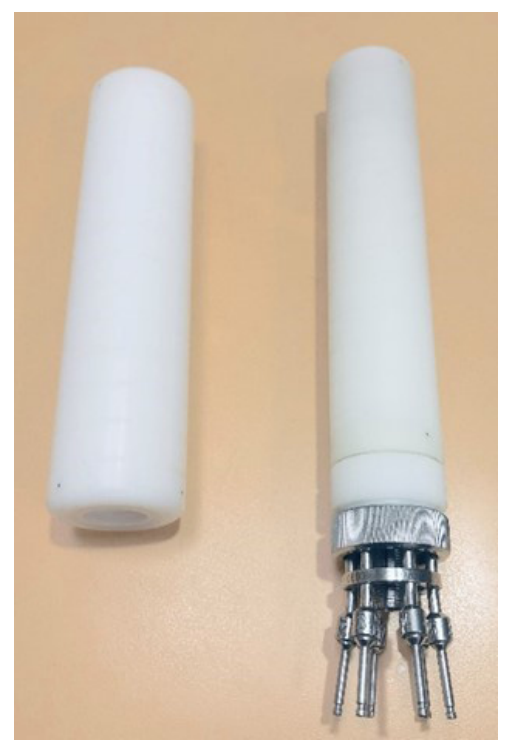

Figure 1. Miami Style applicator set — VARIAN medical systems 


\section{Prescription and planning}

The images were transferred to the treatment planning system (Brachyvision Treatment Planning System, version 13.6 Varian medical systems, Palo Alto, CA). A dose of 5.5-6.5 Gy per fraction for 4 fractions was prescribed $0.5 \mathrm{~cm}$ from the surface of the applicator, amounting to a total Biological Effective Dose (BED) of 34 Gy (Fig. 2). The mean dose at the surface of the vault with this schedule was $7.6 \mathrm{~Gy}$ per fraction. This dose resulted in a total BED of $55.5 \mathrm{~Gy}$ at the surface. The length prescribed varied depending on the histology, but in all patients a minimum of an upper $3 \mathrm{~cm}$ was included in the treatment volume. The inferior extent of vaginal treatment length was increased in patients having serous or clear cell histology or endometrial sarcoma.

\section{Treatment and follow-up}

All patients were treated with a Gamma-med Plus iX (Varian medical systems, Palo Alto, CA) remote after a loader with an Ir 192 source. Subsequent applications were done similarly after 3-7 days. Patients did not undergo CT simulation for the remaining fractions, and the same plan was executed.

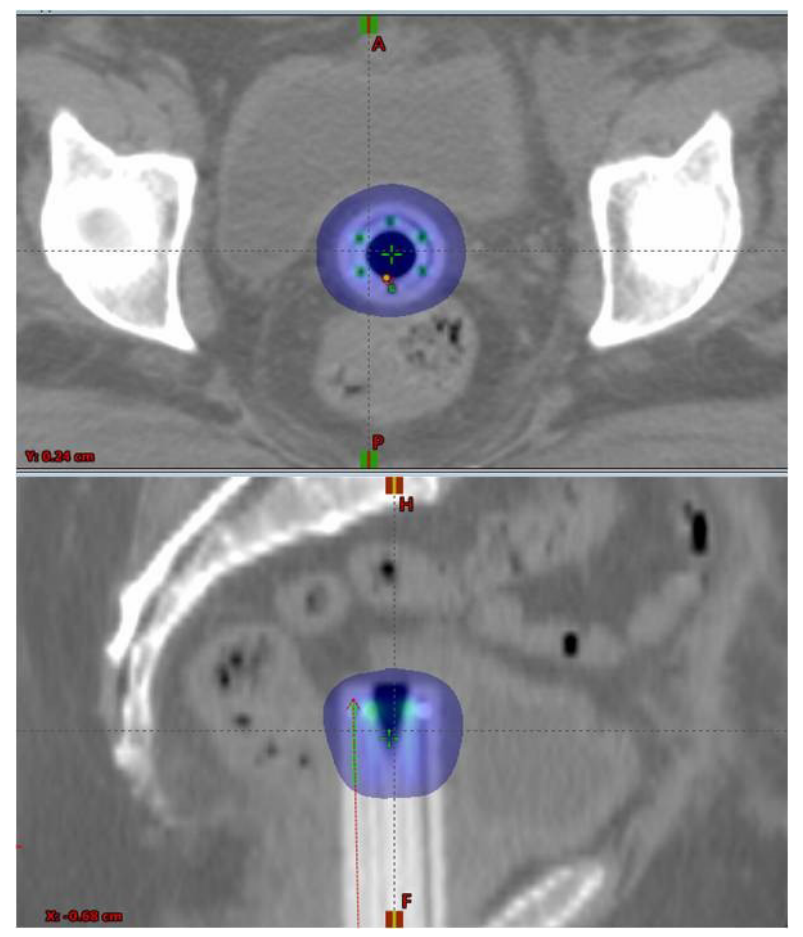

Figure 2. Dose distribution using the Miami Style applicator set
After completion of brachytherapy, patients were followed up every 2 months for 2 years, every 3 months for the third year and 6 months thereafter and annually after 5 years. They underwent a clinical examination only at each follow-up. Imaging was reserved for symptomatic patients or patients with suspected recurrence. All recurrences were proven by biopsy. Treatment failures were classified as local recurrence, loco-regional recurrence or distant metastases.

\section{Results}

\section{Patient demographics}

A total of 62 patients treated between May 2013 and Jan 2018 and with a minimum follow-up of 6 months were included in this analysis. The average age of the patients was $61 \mathrm{yrs}$ (range 36-85 yrs) (Tab. 1).

Out of 62 patients, 60 presented with postmenopausal bleeding, 1 was operated upon for leiomyoma and 1 for prolapse with pelvic inflammatory disease.

Total abdominal hysterectomy with bilateral salpingo-oophorectomy with pelvic lymph node dissection was performed in $97 \%$ (60 out of 62) of the patients. The remaining underwent a simple hysterectomy for a presumed benign condition and were later diagnosed to have malignancy on his-

Table 1. Patient demographics

\begin{tabular}{|l|c|}
\hline Mean age & 61 yrs (range 36-85 yrs) \\
\hline Histology & $54(84 \%)$ \\
\hline Adenocarcinoma & $4(6 \%)$ \\
\hline $\begin{array}{l}\text { Adenocarcinoma with squamous } \\
\text { metaplasia }\end{array}$ & $1(2 \%)$ \\
\hline Clear cell & $1(2 \%)$ \\
\hline Mixed Müllerian malignant tumor & $1(2 \%)$ \\
\hline Endometrial stromal sarcoma & $1(2 \%)$ \\
\hline Papillary serous & \\
\hline Stage & $22(35 \%)$ \\
\hline IA & $36(58 \%)$ \\
\hline IB & $4(6.4 \%)$ \\
\hline II & $34(55 \%)$ \\
\hline Dose & $25(40 \%)$ \\
\hline 5.5 GY & $3(05 \%)$ \\
\hline 6 Gy & \\
\hline 6.5 Gy & \\
\hline
\end{tabular}


Table 2. Grade of tumour in different stages

\begin{tabular}{|l|c|c|c|}
\multicolumn{1}{|c}{} & Grade 1 & Grade 2 & Grade 3 \\
\hline Stage IA & 14 & 3 & 5 \\
\hline Stage IB & 18 & 13 & 5 \\
\hline Stage II & 3 & 1 & 0 \\
\hline & $35(56 \%)$ & $17(27 \%)$ & $10(16 \%)$ \\
\hline
\end{tabular}

topathological analysis. None of the patients had a positive margin, and all the resected pelvic lymph nodes were negative. The average number of lymph nodes resected was 14 (range 5-40).

Histologically, out of 62 patients, 52 (84\%) had endometroid adenocarcinoma. The less frequent histologies were adenocarcinoma with squamous differentiation, malignant mixed mullerian tumor, clear cell and papillary serous adenocarcinoma and endometrial stromal sarcoma.

When classified by stage, 22 (35\%) patients had stage IA disease, 36 (58\%) patients had stage IB and $4(6.4 \%)$ patients had stage II endometrial cancer. Among the stage IA and IB patients, the majority were well differentiated tumors (Tab. 2).

The dose prescribed was in the range of 5.5-6.5 Gy per fraction for 4 fractions (Tab. 1).

At a median follow-up of 19 months (6-48 months), 58 (93\%) patients were alive and well without disease.

\section{Local recurrence}

Two patients had vault only recurrence. One patient had local recurrence at 8 months for which salvage chemotherapy and radiotherapy was given. She progressed to have distant metastases at 16 months to the liver, lung and bones and expired at 16 months. The second patient recurred locally at 17 months, and salvage chemotherapy was provided. She was alive and without disease at the last follow-up. There were no specific histopathologic features that could be attributed to the recurrence in these patients. There was no underdosing of the vault in the patients who had local recurrence.

\section{Loco-regional recurrence}

One patient had metastases to the paracaval, paraaortic and common iliac nodes causing obstructive renal failure at 31 months. She was not fit for any treatment due to a poor performance status and eventually succumbed to the disease within a week of diagnosis.

\section{Metastatic disease}

One patient had progressive metastatic disease during treatment. She was diagnosed to have malignant ascites after the 3rd fraction of brachytherapy. She was planned for palliative chemotherapy, but there was a rapid progression of the disease and she expired.

One patient had biopsy-proven bone metastases to the third lumbar vertebra with cord compression at 17 months for which surgical decompression was performed followed by palliative radiotherapy and palliative chemotherapy. The patient was alive and well on maintenance hormone [megestrol acetate] therapy at last follow-up.

One patient had metachronous carcinoma of the breast diagnosed 4 months after completion of treatment for endometrial cancer. She was treated with surgery but progressed to have multiple brain metastases within a year and succumbed to metastatic breast cancer.

None of the patients had any grade 2 or higher vaginal, gastrointestinal or genitourinary toxicity.

\section{Discussion}

We present the results of 62 patients treated with vaginal brachytherapy alone using a multichannel vaginal applicator for early-stage endometrial cancer. Adjuvant treatment for early-stage endometrial cancer still remains controversial despite the good body of literature published. Available treatment options include pelvic external beam radiotherapy alone, pelvic radiotherapy with brachytherapy boost and vaginal brachytherapy alone.

Three trials evaluated the role of external radiotherapy in intermediate risk endometrial cancer [4-6]. The trials showed that pelvic radiotherapy was associated with lower relapses, but there was no difference in overall survival. External radiotherapy was shown to be associated with significant gastrointestinal and genitourinary toxicities.

The PORTEC-2 trial was conducted to establish if vaginal brachytherapy alone could provide similar benefit with reduced toxicity [8]. The trial showed similar results in terms of overall survival, disease-free survival and vaginal, loco-regional and distant recurrences compared with external beam radiotherapy but with reduced toxicity. Following the results of the PORTEC-2 trial, other studies demonstrated similar results [9-11]. Vagi- 
nal brachytherapy, therefore, was preferred over external beam radiotherapy in intermediate risk endometrial cancer.

We demonstrated 93\% loco-regional control with vaginal brachytherapy alone in our cohort of patients, which included both intermediate- and high-risk patients. We could not identify any particular histopathologic features for relapse in the 2 patients who had local failure and 1 patient who failed in the pelvis. Of the 2 patients who failed locally, one was disease free at the time of analysis with salvage treatment.

There is no clear consensus to define patients as low and high in the intermediate-risk group with varying criteria among the different studies. The recommendations are equally ambiguous for adjuvant radiotherapy. The PORTEC trials recommended observing those women who fall in the low intermediate-risk group, whereas the NCCN recommends either observation or vaginal brachytherapy $[4,8,12]$. The NCCN even includes external beam radiotherapy as part of the management for the high intermediate-risk group even as the PORTEC-2 trial favored brachytherapy alone. Our preference has been to offer adjuvant vaginal brachytherapy to all patients except those women who have a Grade 1 disease confined to the endometrium only. We do not offer external beam radiotherapy for any stage I patients due to the associated toxicity. Long-term outcomes of patients enrolled in the TME, PORTEC- 1 and 2 trials showed increased bowel toxicity and limitation of daily activities in the pelvic radiotherapy arm but no evidence that EBRT increased the risk of second neoplasms $[13,14]$. There were no differences in overall or failure-free survival. Patients who received vaginal brachytherapy alone had better QOL [14]. In this study, there was only one patient who failed in the pelvis, and the majority had distant recurrences. With low pelvic-only failures, external radiotherapy can be safely omitted in stage I patients.

Various applicators are available for delivering vaginal brachytherapy. Single channel vaginal cylinders are the preferred choice in many centers. Possibilities for dose optimization have been a limitation with single channel applicators. Multichannel applicators were developed as a bridge between single channel vaginal applicators and the more invasive interstitial implant. These applicators are particularly useful for circular tumor geometry or in the adjuvant setting where dose can be reduced to the vaginal mucosa, bladder and rectum $[15,16]$. Demanes et al. used California's Endocurietherapy multichannel vaginal cylinder and showed that by not loading the 12 oclock and 6 oclock peripheral catheters, the dose distribution could be flattened, thus reducing the dose to the bladder and rectum [15]. At our institute, we use the Miami multichannel vaginal applicator. It offers a means to optimize the dose based on patient anatomy. An asymmetric dose distribution along the axis of the cylinder can be generated and the dose to critical structures can be minimized [17]. There are limited data on whether this dosimetric advantage actually translates into significant clinical outcomes. We, therefore, do not prescribe any dose constraints to the bladder and rectum.

The Miami kit contains a cylindrical obturator with 6 peripheral channels and a central channel that can accommodate a straight or curved tandem that can be used to treat the disease in the cervix. It also has build-up caps with diameters of $3 \mathrm{~mm}$, $3.5 \mathrm{~mm}$ and $4 \mathrm{~mm}$. The $3 \mathrm{~mm}$ diameter build-up cap was placed in almost all our cases. This cap was likely used because of the smaller pelvis and narrow vagina seen in most Indian women. A single treatment plan was generated for all the fractions using CT simulation images obtained before the first HDR treatment with the Miami applicator in place. The position of the applicator was verified, and subsequent fractions were delivered with the same plan.

There is no single universally accepted dose fractionation regime for adjuvant treatment with brachytherapy alone. The ABS recommends delivering approximately 60 Gy low dose rate (LDR) equivalent to the vaginal surface (ABS) [18]. Various institutions have implemented different fractionation schemes with acceptable outcomes. Using $7 \mathrm{~Gy} \times 3$ fractions prescribed at $0.5 \mathrm{~cm}$ or $6 \mathrm{~Gy} \times 5$ fractions or $4 \mathrm{~Gy} \times 6$ fractions prescribed to the surface are some of the common dose fractionation schedules [18]. Our preferred schedule has been 5.5-6 Gy $\times 4$ fractions prescribed at $0.5 \mathrm{~cm}$ from the surface, which achieves similar LDR equivalent doses as recommended.

Gerbhardt et al. have demonstrated that using a multichannel vaginal cylinder with image guidance can result in high local control and low rates of severe toxicity [19]. There was no Grade 2 or 
higher bladder or rectal toxicity in any of our patients, and our local recurrence rate was on par with published results. We, therefore, believe in imageguided planning for adjuvant brachytherapy alone, which conforms the dose to the target region and minimizes long-term toxicity.

Our local recurrence rate of 3\% (2 out of 62 patients) with only 1 regional failure is on par with, if not better than, outcomes from other centers. None of the recurrences had any correlation with the stage or grade of the disease. We had no increased recurrences with brachytherapy alone for the defined high-risk subsets, such as Ib grade 3, stage II or the papillary serous and clear cell varieties, but our numbers are very small. There is sufficient evidence in the literature that high-risk endometrial cancer (Ib/Ic and grade 3 ) treated with brachytherapy alone showed low local or pelvic recurrence [20-23]. Rasool et al. concluded that patients with stage IB/IC, grade 3 (IA/IB as per recent staging) endometroid adenocarcinoma had a significant risk for extrapelvic recurrence, and there was no significant difference in recurrence based on treatment modality [24]. Desai et al. studied stage I-II uterine papillary serous carcinoma and concluded that the risk of isolated pelvic recurrence was too low to warrant a routine use of external radiotherapy [25]. Matsuo et al. noticed an association between the extent of pelvic lymphadenectomy and the use of vaginal brachytherapy. Their results showed that there was a trend in counselling patients for brachytherapy alone if $>10$ nodes were dissected [26].

More data are needed before suggesting that external radiotherapy can be safely omitted in patients with high-risk stage I endometrial cancers.

Standard definitions for target volume delineation and dose constraints for OARs are necessary for uniform incorporation of image-guided brachytherapy across centers. Similarly, establishing guidelines for adjuvant radiotherapy is needed to identify the subset of patients who will absolutely benefit from external radiotherapy.

The limitations of our study include its retrospective design and lack of dose constraints to the organs at risk. We did not have any patient with grade 2 or higher toxicity, but there was probable underreporting of grade 1 toxicity. Despite this limitation, we have been successful in reporting a large number of patients in whom external radiotherapy was avoided with low recurrence rates.

\section{Conclusion}

Vaginal brachytherapy alone should be considered for all stage I and selected stage II patients as it provides excellent local control with very low toxicity. For the high-risk subset, larger numbers are needed before recommending avoiding external beam radiation. Image-guided brachytherapy allows for conformal dose distribution, and the use of a multichannel applicator can potentially minimize the dose to the organs at risk. Prospective randomized data are required to validate these findings.

\section{Conflicts of interest}

There are no relevant conflicts of interest.

\section{Funding}

The authors confirm that neither we nor our institution at any time receive payment or services from a third party for any aspect of the submitted work.

\section{Data sharing statement}

All data generated and analyzed during this study are included in this published article.

\section{Authors' contribution}

K.S.R. - primary author and treating radiation onologist; S.D. - data compilation and follow up; M.P. - treating radiation oncologist; P.R. - treating radiation oncologist; G.G.V. - treating radiation oncologist; A.V. - operating surgeon; S.B.S. - operating surgeon.

\section{References}

1. Siegel RL, Miller KD, Jemal A. Cancer statistics, 2018. CA Cancer J Clin. 2018; 68(1): 7-30, doi: 10.3322/caac.21442, indexed in Pubmed: 29313949.

2. Maheshwari A, Kumar N, Mahantshetty U. Gynecological cancers: A summary of published Indian data. South Asian J Cancer. 2020; 05(03): 112-120, doi: 10.4103/2278330x.187575, indexed in Pubmed: 27606294.

3. Kong A, Johnson N, Kitchener $\mathrm{HC}$, et al. Adjuvant radiotherapy for stage I endometrial cancer: an updated Cochrane systematic review and meta-analysis. J Natl Cancer Inst. 2012; 104(21): 1625-1634, doi: 10.1093/jnci/ djs374, indexed in Pubmed: 22962693.

4. Nout RA, van de Poll-Franse LV, Lybeert MLM, et al. PORTEC Study Group. Long-term outcome and quality of life of patients with endometrial carcinoma treated with or without pelvic radiotherapy in the post operative radiation therapy in endometrial carcinoma 1 (PORTEC-1) trial. J Clin Oncol. 2011; 29(13): 1692-1700, doi: 10.1200/ JCO.2010.32.4590, indexed in Pubmed: 21444867. 
5. Blake P, Swart AM, Orton J, et al. ASTEC/EN.5 Study Group. Adjuvant external beam radiotherapy in the treatment of endometrial cancer (MRC ASTEC and NCIC CTG EN.5 randomised trials): pooled trial results, systematic review, and meta-analysis. Lancet. 2009; 373(9658): 137-146, doi: 10.1016/S0140-6736(08)61767-5, indexed in Pubmed: 19070891.

6. Keys HM, Roberts JA, Brunetto VL, et al. Gynecologic Oncology Group. A phase III trial of surgery with or without adjunctive external pelvic radiation therapy in intermediate risk endometrial adenocarcinoma: a Gynecologic Oncology Group study. Gynecol Oncol. 2004; 92(3): 744-751, doi: 10.1016/j.ygyno.2003.11.048, indexed in Pubmed: 14984936.

7. McCloskey SA, Tchabo NE, Malhotra HK, et al. Adjuvant vaginal brachytherapy alone for high risk localized endometrial cancer as defined by the three major randomized trials of adjuvant pelvic radiation. Gynecol Oncol. 2010; 116(3): 404-407, doi: 10.1016/j.ygyno.2009.06.027, indexed in Pubmed: 19944453.

8. Nout RA, Smit VT, Putter H, et al. PORTEC Study Group. Vaginal brachytherapy versus pelvic external beam radiotherapy for patients with endometrial cancer of high-intermediate risk (PORTEC-2): an open-label, noninferiority, randomised trial. Lancet. 2010; 375(9717): 816-823, doi: 10.1016/S0140-6736(09)62163-2, indexed in Pubmed: 20206777.

9. Kellas-Ślęczka S, Wojcieszek P, Białas B. Educational Corner Adjuvant vaginal brachytherapy as a part of management in early endometrial cancer. J Contemp Brachyther. 2012; 4: 247-252, doi: 10.5114/jcb.2012.32560.

10. Jolly $S$, Vargas $C$, Kumar $T$, et al. Vaginal brachytherapy alone: an alternative to adjuvant whole pelvis radiation for early stage endometrial cancer. Gynecol Oncol. 2005; 97(3): 887-892, doi: 10.1016/j.ygyno.2005.02.021, indexed in Pubmed: 15943991.

11. Lin LL, Mutch DG, Rader JS, et al. External radiotherapy versus vaginal brachytherapy for patients with intermediate risk endometrial cancer. Gynecol Oncol. 2007; 106(1): 215-220, doi: 10.1016/j.ygyno.2007.03.024, indexed in Pubmed: 17482665.

12. NCCN Clinical Practice Guidelines. Uterine Neoplasms. Version 2.2018, May 2018.

13. Creutzberg CL, Nout RA, Lybeert MLM, et al. PORTEC Study Group. Fifteen-year radiotherapy outcomes of the randomized PORTEC-1 trial for endometrial carcinoma. Int J Radiat Oncol Biol Phys. 2011;81(4): e631-e638, doi: 10.1016/j. ijrobp.2011.04.013, indexed in Pubmed: 21640520.

14. Nout RA, Putter $H$, Jürgenliemk-Schulz IM, et al. Quality of life after pelvic radiotherapy or vaginal brachytherapy for endometrial cancer: first results of the randomized PORTEC-2 trial. J Clin Oncol. 2009; 27(21): 3547-3556, doi: 10.1200/JCO.2008.20.2424, indexed in Pubmed: 19546404.

15. Demanes D, Rege $S$, Rodriquez $R$, et al. The use and advantages of a multichannel vaginal cylinder in high-dose-rate brachytherapy. Int J Radiat Oncol Biol Phys. 1999; 44(1): 211-219, doi: 10.1016/s0360-3016(98)00453-2, indexed in Pubmed: 10219816.
16. Bahadur YA, Constantinescu C, Hassouna AH, et al. Single versus multichannel applicator in high-doserate vaginal brachytherapy optimized by inverse treatment planning. J Contemp Brachytherapy. 2015; 6(4): 362-370, doi: 10.5114/jcb.2014.47816, indexed in Pubmed: 25834580.

17. Iftimia I, Cirino ET, Mower HW, et al. Treatment planning methodology for the Miami Multichannel Applicator following the American Brachytherapy Society recently published guidelines: the Lahey Clinic experience. J Appl Clin Med Phys. 2013; 14(1): 4098, doi: 10.1120/jacmp. v14i1.4098, indexed in Pubmed: 23318396.

18. Small W, Beriwal S, Demanes DJ, et al. American Brachytherapy Society. American Brachytherapy Society consensus guidelines for adjuvant vaginal cuff brachytherapy after hysterectomy. Brachytherapy. 2012; 11(1): 58-67, doi: 10.1016/j.brachy.2011.08.005, indexed in Pubmed: 22265439.

19. Gebhardt BJ, Vargo JA, Kim H, et al. Image-based multichannel vaginal cylinder brachytherapy for the definitive treatment of gynecologic malignancies in the vagina. Gynecol Oncol. 2018; 150(2): 293-299, doi: 10.1016/j. ygyno.2018.06.011, indexed in Pubmed: 29929925.

20. Chadha M, Nanavati PJ, Liu P, et al. Patterns of failure in endometrial carcinoma stage IB grade 3 and IC patients treated with postoperative vaginal vault brachytherapy. Gynecol Oncol. 1999; 75(1): 103-107, doi: 10.1006/ gyno.1999.5526, indexed in Pubmed: 10502434.

21. Anderson J, Stea B, Hallum A, et al. High-dose-rate postoperative vaginal cuff irradiation alone for stage $\mathrm{lb}$ and Ic endometrial cancer. Int J Radiat Oncol Biol Phys. 2000; 46(2): 417-425, doi: 10.1016/s0360-3016(99)00427-7, indexed in Pubmed: 10661349.

22. Rittenberg PVC, Lotocki RJ, Heywood MS, et al. High-risk surgical stage 1 endometrial cancer: outcomes with vault brachytherapy alone. Gynecol Oncol. 2003; 89(2): 288-294, doi: 10.1016/s0090-8258(03)00085-4, indexed in Pubmed: 12713993.

23. Rittenberg PVC, Lotocki RJ, Heywood MS, et al. Stage II endometrial carcinoma: limiting post-operative radiotherapy to the vaginal vault in node-negative tumors. Gynecol Oncol. 2005; 98(3): 434-438, doi: 10.1016/j. ygyno.2005.04.030, indexed in Pubmed: 16005498.

24. Rasool N, Fader A, Seamon L, et al. Stage I, grade 3 endometrioid adenocarcinoma of the endometrium: An analysis of clinical outcomes and patterns of recurrence. Gynecol Oncol. 2010; 116(1): 10-14, doi: 10.1016/j. ygyno.2009.10.043, indexed in Pubmed: 19875158.

25. Desai NB, Kiess AP, Kollmeier MA, et al. Patterns of relapse in stage I-II uterine papillary serous carcinoma treated with adjuvant intravaginal radiation (IVRT) with or without chemotherapy. Gynecol Oncol. 2013; 131(3): 604-608, doi: 10.1016/j.ygyno.2013.09.019, indexed in Pubmed: 24055615.

26. Matsuo K, Machida H, Ragab OM, et al. Extent of pelvic lymphadenectomy and use of adjuvant vaginal brachytherapy for early-stage endometrial cancer. Gynecol Oncol. 2017; 144(3): 515-523, doi: 10.1016/j. ygyno.2016.12.012, indexed in Pubmed: 28017306. 